\title{
CHARACTERIZATION OF DEFECT CENTRES IN SEMICONDUCTORS BY ADVANCED ENDOR TECHNIQUES
}

\author{
T. Gregorkiewicz, H.E. Altink and C.A.J. Ammerlaan \\ Van der Waals-Zeeman Laboratorium, Universiteit van Amsterdam \\ Valckenierstraat 65, 1018 XE Amsterdam, The Netherlands
}

The advanced magnetic resonance techniques and their application to the studies of defects in semiconductors will be reviewed. Transient and stationary ENDOR, optically detected ENDOR and double ENDOR variations will be briefly discussed while special attention will be given to the Field-Stepped-ENDOR technique. The successful application of the advanced ENDOR techniques for the structure determination of complex defects will be illustrated by the examples concerning the boron-vacancy complex and thermal donors in silicon and the gallium vacancy in gallium phosphide.

PACS numbers: $61.16 . \mathrm{Hn}$

\section{Introduction}

Ever since its discovery by Zavoisky in 1944 electron paramagnetic resonance (EPR), has proven itself extremely useful in the identification and structural studies of paramagnetic centres. Later, with the development of semiconductor physics and materials science, EPR has found its permanent place as one of the most prominent experimental techniques. The apparent success EPR owes to its unique capability of providing information on atomic scale. In fact the microscopic structural models of all the most important defect centres in modern electronic materials were developed on the basis of magnetic resonance data.

Magnetic resonance technique deals with the observations of the interaction of the total magnetic moment with the external magnetic field. Due to the rather big energy separation between the spin-split energy levels (in the $\mathrm{GHz}$ range) the EPR method is rather sensitive but for the same reason its energetic resolution is limited. It is this particular handicap of the EPR that led to the development of the double resonance technique known as ENDOR, i.e. Electron Nuclear Double 
Resonance. In this method the transition induced between the levels split due to the interaction with the nuclear magnetic moment are detected by their influence on the simultaneously induced EPR transition. In this way the high sensitivity of the EPR is combined with the high resolving power of nuclear magnetic resonance. In ENDOR experiments the resolution in the range of $\mathrm{kHz}$ is obtained while the sensitivity is usually only one order of magnitude lower than in conventional EPR.

To illustrate the idea of an ENDOR experiment let us consider a simple system with total electron spin $S=1 / 2$ and nuclear spin $I=1 / 2$ (a physical example of such system would be substitutional phosphorus atom in $n$-type silicon). Since both the electron and the nuclear spins can have two orientations with respect to the external magnetic field, there are four possible energy levels as shown in Fig. 1. An EPR transition is governed by the selection rules $\Delta m_{S}= \pm 1, \Delta m_{I}=0$ so

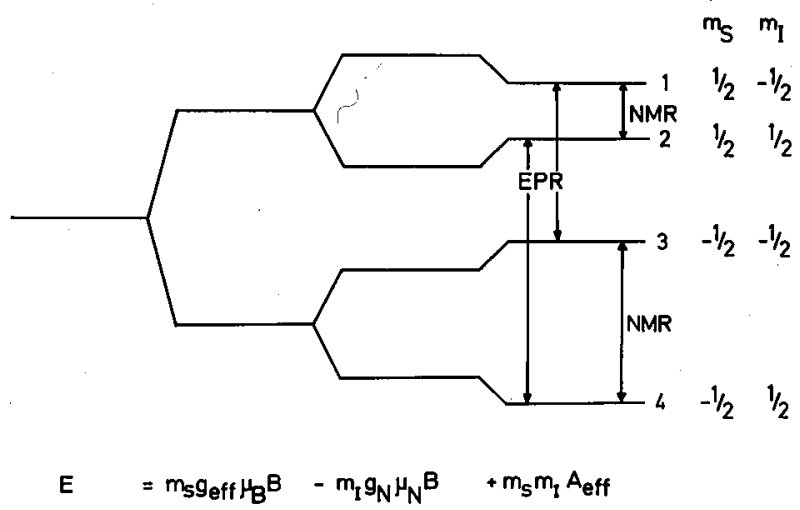

Fig. 1. Energy level scheme for $S=1 / 2, I=1 / 2$ system. The allowed EPR and NMR transitions have been indicated.

that only the electron spin orientation is changed. Therefore, as depicted in Fig. 1, EPR transitions can occur between energy levels 1 and 3 and between 2 and 4. Let us further focus our attention on the EPR transition $1 \leftrightarrow 3$. The intensity of the corresponding EPR line will depend on the population difference between these two energy levels. If, while satisfying the resonance conditions for the $1 \leftrightarrow 3$ transition, one applies to the system also radio frequency radiation which corresponds to the energy difference between levels 3 and 4 or 1 and 2 , then the nuclear magnetic resonance (NMR) transition $\left(\Delta m_{S}=0, \Delta m_{I}= \pm 1\right)$ will be simultaneously induced. As a result the populations of energy levels 3 or 1 will be altered, respectively. This in turn will lead to a transient change of the observed EPR

signal creating the so-called ENDOR response. Through the increase in population difference between 1 and 2 or 3 and 4 caused by the (high-power) EPR transition $1 \leftrightarrow 3$ and because of the bigger quantum energy of the EPR transition compared to the NMR transition, the NMR sensitivity is in ENDOR typically enhanced by five orders of magnitude. The ENDOR technique therefore combines the high sensitivity of EPR with the high resolution of NMR. In addition to the above described transient ENDOR the ENDOR signal can also be observed un- 
der stationary conditions. The idea of stationary ENDOR is illustrated in Fig. 2. The amplitude of the observed EPR signal is determined here on one side by the

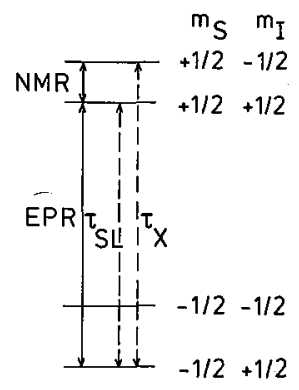

Fig. 2. Stationary ENDOR for $S=1 / 2, I=1 / 2$ system. The available paths for spin-lattice $\tau_{\mathrm{SL}}$ and cross relaxations $\tau_{X}$ have been indicated.

amount of microwave power being delivered into the system and on the other side by the relaxation processes responsible for the dissipation of the energy within the system. The principal relaxation path via spin-lattice coupling $\tau_{\mathrm{SL}}$ is indicated. When an NMR transition is simultaneously induced linking the $m_{I}=+1 / 2$ and $m_{I}=-1 / 2$ levels new relaxation mechanisms become available, $\tau_{X}$, leading to a permanent change of the EPR signal. The actual choice of the suitable ENDOR technique depends on the system; Fig. 3 presents the comparison of transient and stationary ENDOR techniques as applied to the same system of the arsenic donor in silicon $[1,2]$.

Since in the ENDOR experiment the NMR transitions are being induced it follows naturally that the technique is suitable for determination of the hyperfine interactions, i.e. the interactions between electron moment $J$ (often treated as effective spin) and nuclear moment $I$. Such interaction can be with the nuclear moment of the centre itself as well as with the nuclear spins of the nuclei of the surrounding crystal. In this sense the division into so-called self- and ligand-ENDOR is introduced. In case of some paramagnetic centres the hyperfine interactions are big and as such can be visible already in EPR allowing, in most cases, for the identification of the nuclei involved - good examples are here again shallow donors in silicon or the Si:Ti system. Performing self-ENDOR of such centres can confirm this identification allowing at the same time considerable insight into the electron configuration, core polarization and covalency effects [3] while in a ligand-ENDOR experiment the spin distribution on the neighbouring atomic shells can be determined unravelling the details of the electronic wave function character (spin mapping) [4]. Figure 4 presents the EPR spectrum of one of the newly discovered Zn-related EPR centres in silicon [5]. The spectrum of the centre, identified as zinc-chromium complex, is presented for two different orientations of the magnetic field $\boldsymbol{B}$. The involvement of chromium can easily be concluded from clearly visible hyperfine splitting (4 equidistant lines). At the same time a much smaller hyperfine interaction can also be observed as two or four (depending on the angle) small intensity components very close to the central resonance. This splitting arises most 


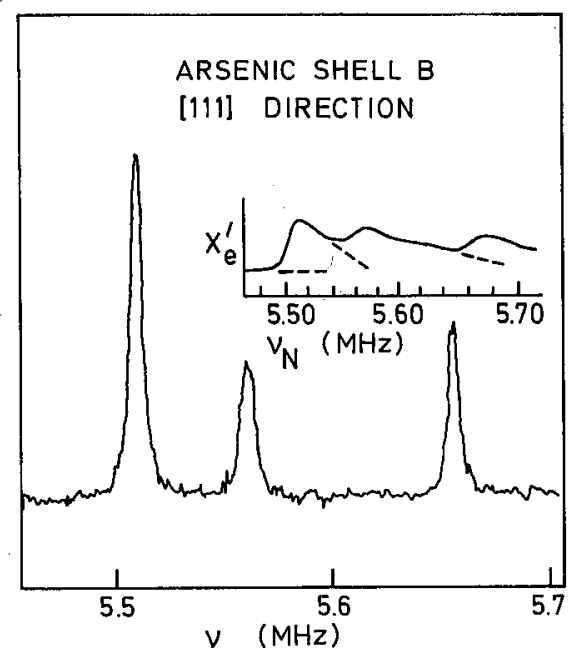

Fig. 3. The comparison of transient [1] (inset) and stationary [2] ENDOR spectra for arsenic donor in silicon.

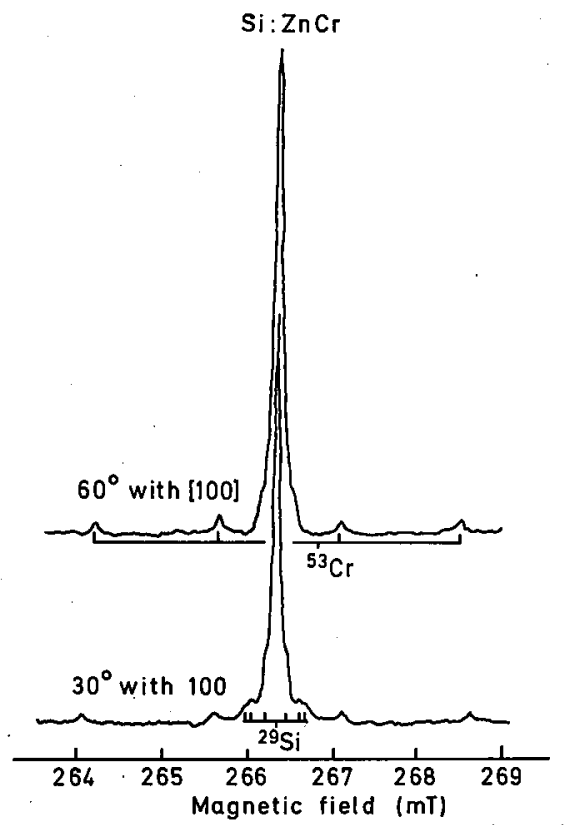

Fig. 4. EPR spectra for $\mathrm{ZnCr}$ complex in silicon [5] as measured in $\mathrm{X}$ microwave band for two different orientations of the magnetic field. The hyperfine structure due to chromium and silicon is indicated. 
probably from the interaction with (nearest) silicon shells and its further analysis by ENDOR would be very helpful in constructing the microscopic model of this particular impurity complex.

In addition to the "standard" ENDOR as outlined above several more advanced modifications of this technique have been developed in order to cope with specific requirements of the problems encountered in materials science of semiconductors. The most widely known is optically detected ENDOR - OD-ENDOR which is simply the ENDOR variation of the well established ODMR technique. In what follows the less known advanced ENDOR variations of field swept EN$D O R$ and double ENDOR will be discussed in more detail. Both methods will be illustrated by their examplary applications in the field of semiconductors.

\section{Field Swept/Stepped ENDOR}

\subsection{Outline of concept}

Two important experimental extensions of ENDOR are the Field Swept ENDOR - FSE (also known under the name of ENDOR-induced EPR [6] and the Field Stepped ENDOR - FStENDOR techniques. Both methods are very

similar and basically serve to obtain an image of the specific EPR-line or line-component which is related to a certain ENDOR transition. They can be used to separate partially overlapping resonance signals, originating from different centres, or from different EPR transitions/orientations superimposed in one experimentally observed resonance line.

In order to illustrate the ideas behind the FSE and FStENDOR techniques let us consider the ENDOR experiment on a more complex $S=1 / 2, I=5 / 2$ system (as appropriate for aluminium ENDOR in the Si-NL10 spectrum). In Fig. 5(a) the energy levels of such systems are shown as a function of the magnetic field. The six allowed EPR transitions are indicated. In Fig. 5(b) the EPR spectrum is simulated under the assumption that the splitting due to the hyperfine interaction is resolved in the EPR experiment. In Fig. 5(c) the ENDOR response is simulated for the case when the field is again scanned over the whole EPR range while simultaneously the NMR transition $9 \leftrightarrow 10$ is induced. The NMR frequency is kept on resonance during the magnetic field scan. Such procedure is followed in the FSE technique. In this case the ENDOR response can only be observed on those EPR transitions which involve the energy levels affected by the $9 \leftrightarrow 10 \mathrm{NMR}$ transition. In Fig. 5(d) the FStENDOR technique is illustrated; in this technique the NMR frequency is scanned for discrete magnetic field values. It can be noted that a few scans at different magnetic.field positions reproduce a similar picture as obtained by FSE.

Both FSE and FStENDOR techniques are very similar and the actual choice among them depends on the particular requirements of the individual experiment. Generally one can say that FSE is much faster while FStENDOR offers higher resolving power. Further, for FSE the knowledge of the nucleus involved in the hyperfine interactions is necessary since the $g_{N}$ value is used to calculate the correction of the NMR resonance frequency; in case of FStENDOR this is not necessary as the actual $g_{N}$ value is being itself determined in the experiment. 

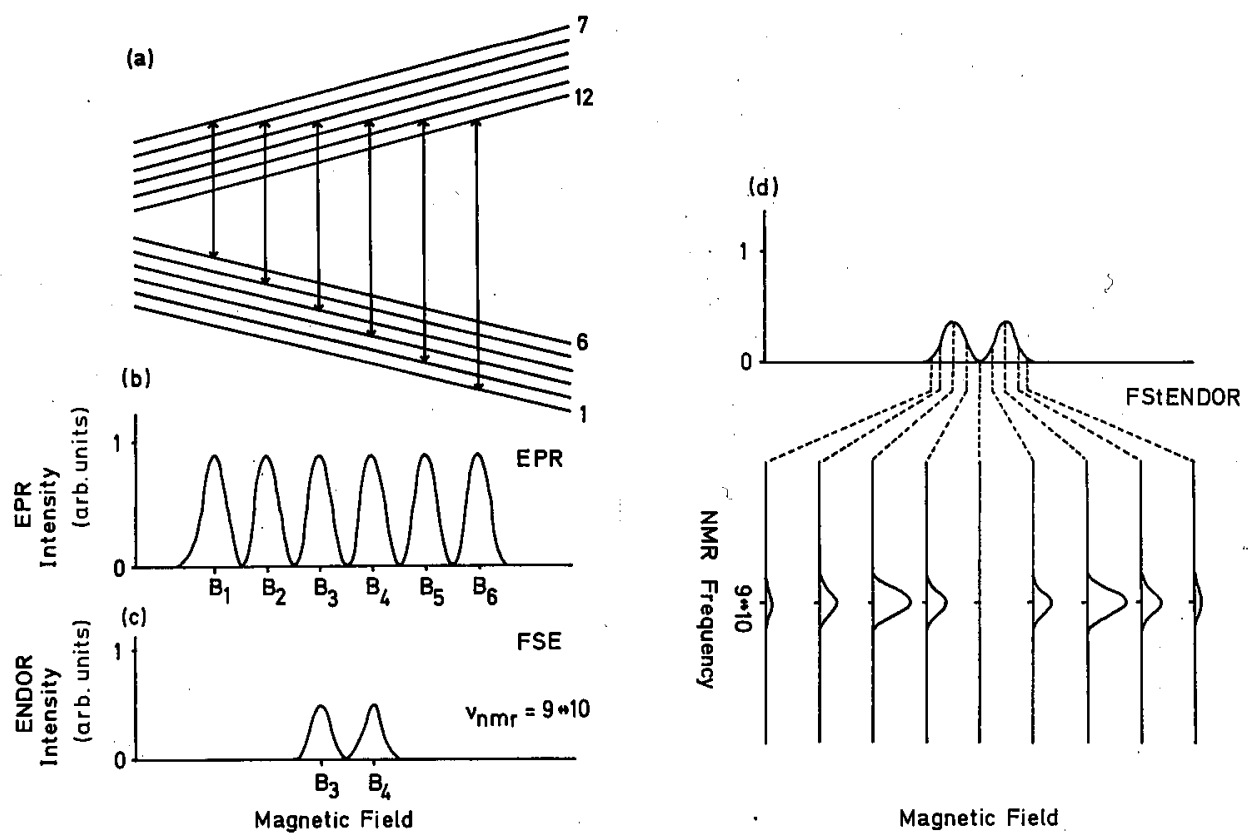

Fig. 5. (a) Energy levels of the $S=1 / 2, I=5 / 2$ system as a function of magnetic field. (b) Simulation of an EPR spectrum for such system under the assumption that the splitting due to the hyperfine interaction is resolved. (c) The ENDOR response when the magnetic field is scanned through the whole range of the EPR spectrum while simultaneously the $9 \leftrightarrow 10$ NMR transition is excited. (d) Simulation of the corresponding FStENDOR spectrum; the NMR frequency is scanned for discrete magnetic field values.

\subsection{Example FSE: Boron-vacancy pair in silicon}

In Fig. 6 a successful application of FSE in case of the boron-vacancy complex in silicon [7] is presented. The boron-vacancy complex with the ${ }^{11} B$ boron isotope represents a $S=1 / 2, I=3 / 2$ system; the hyperfine constant is small and therefore cannot be observed in the EPR spectrum. In the figure the total EPR signal is shown as well as the FSE scans for three different ENDOR lines: $m_{I}=+3 / 2 \leftrightarrow$ $m_{I}=+1 / 2, m_{I}=+1 / 2 \leftrightarrow m_{I}=-1 / 2$, and $m_{I}=-1 / 2 \leftrightarrow m_{I}=-3 / 2$ belonging to different EPR transitions split by the ${ }^{11} B$ hyperfine interaction. As can clearly be seen the FSE technique allows to separate the hyperfine components completely overlapping in the EPR experiment. In this case, in contrast to the simulation of Fig. 5(c), the hyperfine splitting between the two EPR lines for which an ENDOR resonance can be observed is not resolved in the FSE experiment. This results. therefore in three and not four components of the EPR line. Their positions have to be understood as average of the appropriate pairs of the hyperfine structure lines: $|-1 / 2,3 / 2\rangle \leftrightarrow|1 / 2,3 / 2\rangle$ and $|-1 / 2,1 / 2\rangle \leftrightarrow|1 / 2,1 / 2\rangle,|-1 / 2,1 / 2\rangle \leftrightarrow$ $|1 / 2,1 / 2\rangle$ and $|-1 / 2,-1 / 2\rangle \leftrightarrow|1 / 2,-1 / 2\rangle$, and $|-1 / 2,-1 / 2\rangle \leftrightarrow|1 / 2,-1 / 2\rangle$ and $|-1 / 2,-3 / 2\rangle \leftrightarrow|1 / 2,-3 / 2\rangle$ transitions, respectively. 

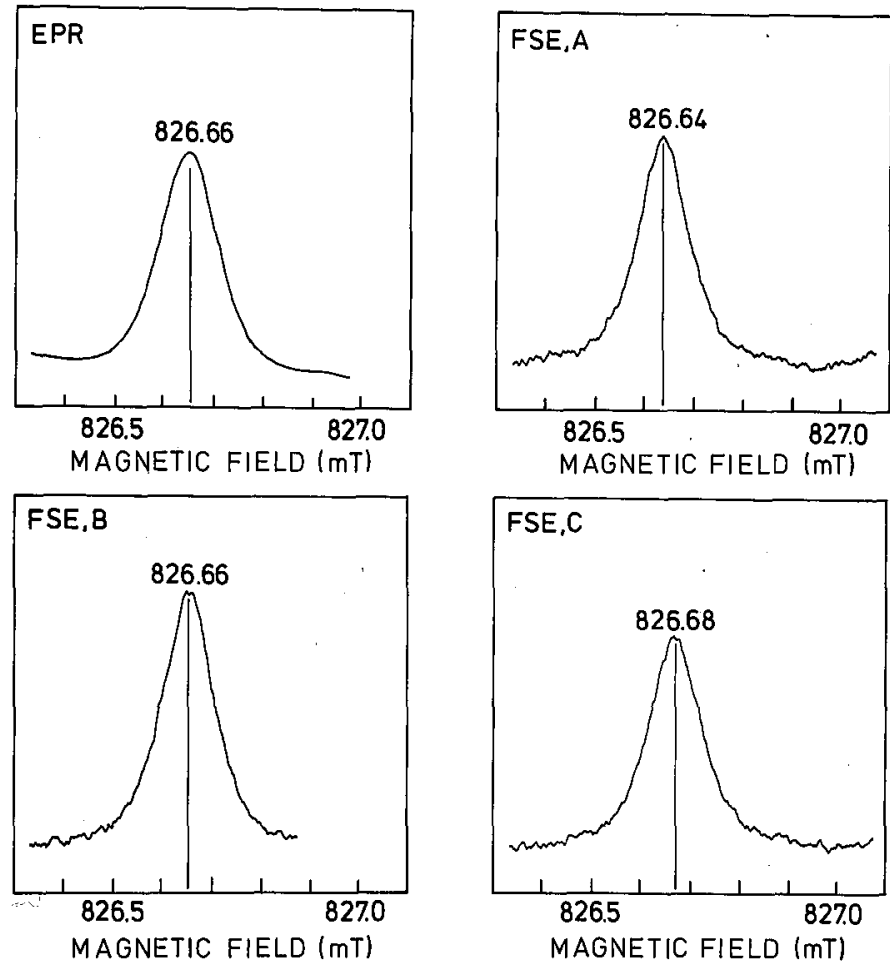

Fig. 6. Successful application of the FSE technique for the boron-vacancy complex in silicon [7]. The hyperfine splitting is not resolved. The total EPR spectrum is compared to the three FSE images obtained for three different ENDOR lines denoted A, B and C.

\subsection{Example FSIENDOR: Thermal donor in silicon}

Thermal donors in silicon constitute a particularly difficult case of prominent defect centre in electronic material. The major difficulty follows here from the fact that there exists a variety of very similar but nevertheless different thermal donors (multispecies character) whose individual EPR spectra are mutually superimposed resulting merely in resonance line broadening and giving rise to the so-called $g$-shifting effect. However individual thermal donor spectra can be resolved in a FStENDOR experiment which is illustrated in Fig. 7 [8]. In the figure three ENDOR scans are depicted; these were performed for three somewhat different values of the magnetic field: at the centre of the EPR line, $B_{\max }$, and of both sides of it. As can clearly be seen the ENDOR lines which belong to different thermal donor species marked 1 to 8 attain their maximum intensity for different magnetic field values. In a similar but more elaborate procedure up to $10 \mathrm{com}-$ ponents of the overall EPR line could be resolved indicating the existence of 10 different thermal donor species. 


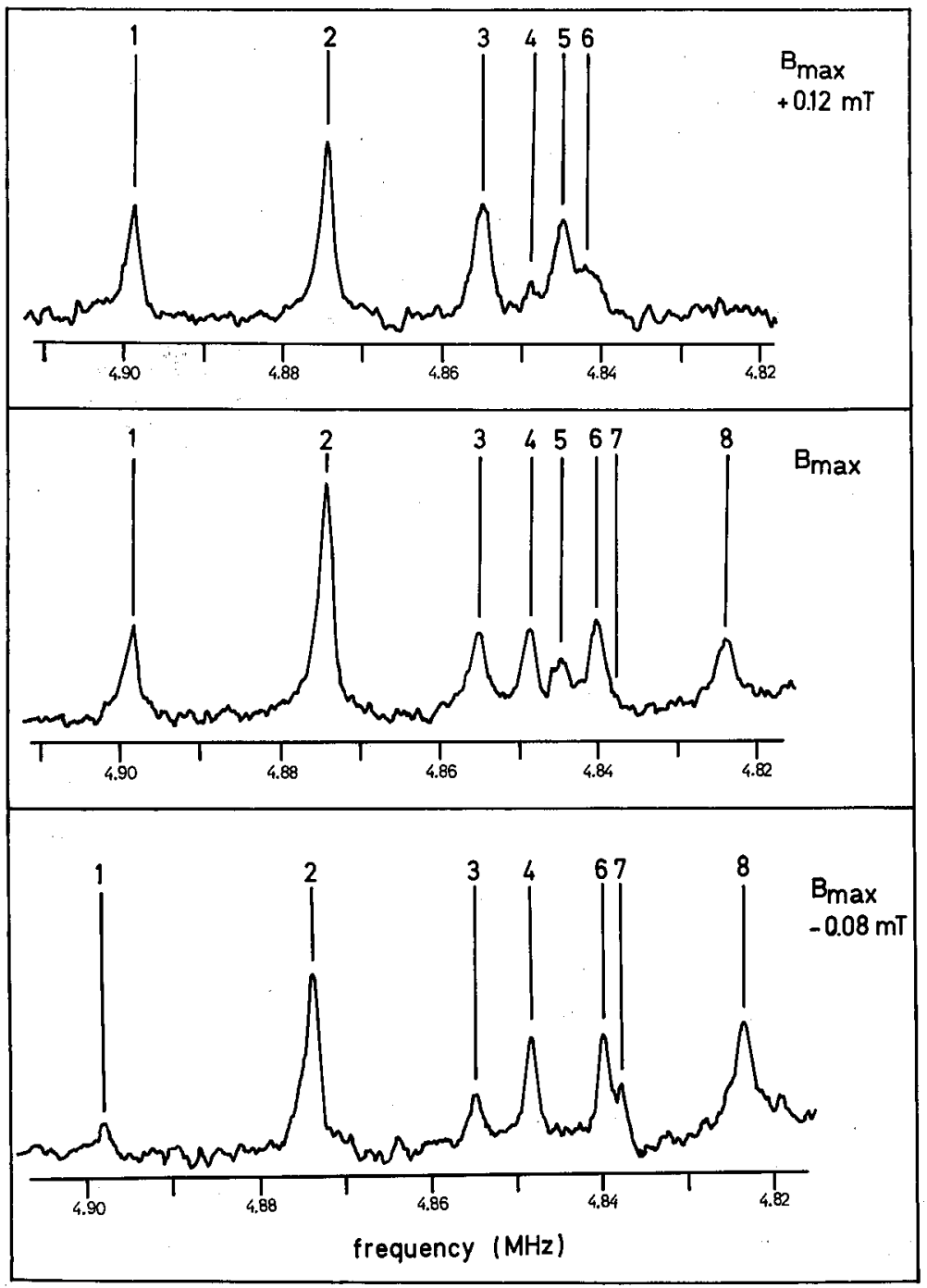

Fig. 7. The illustration of the FStENDOR effect for thermal donor centres in silicon. Numbers 1-8 correspond to different species. The magnetic field is in the [011] direction and $B_{\max }$ corresponds to the magnetic field position in the centre of the EPR line.

\section{Double ENDOR}

\subsection{Outline of concept}

The FSE and FStENDOR techniques allow to increase the EPR resolution and unravel the structure superimposed in the inhomogeneously broadened reso- 
nance line. The Double ENDOR technique (or triple resonance) [9] allows for an immediate identification of the ENDOR lines belonging to one and the same system. Therefore the method is particularly useful when investigating paramagnetic systems with identical EPR spectra which generate different ENDOR spectra. The idea is based here on a rather basic notion that the amplitute of the particular ENDOR signal will be affected if an additional NMR transition within the same system will simultaneously be induced. It can further be shown that the amplitude of the ENDOR response will be reduced or enhanced if the second NMR transition takes place within the same or different $m_{S}$ multiplet, respectively. In experimental practice, where the ENDOR signal is usually detected by modulating the rf source and following the corresponding change of the EPR signal with the lock-in technique, in case of a triple resonance a second (modulated) $\mathrm{rf}$ is introduced and the response of the ENDOR signal is being observed. The effect will only be observed in case that two NMR transitions of the same system are induced while there will be no response for the NMR transitions from different centres (and therefore also not from different orientations of the same complex).

\subsection{Example: Ga vacancy in $G a P$}

The study of the Ga vacancy in GaP by Hage et al.[10] presents an example of successful application of the triple resonance technique. In Fig. 8 an ENDOR
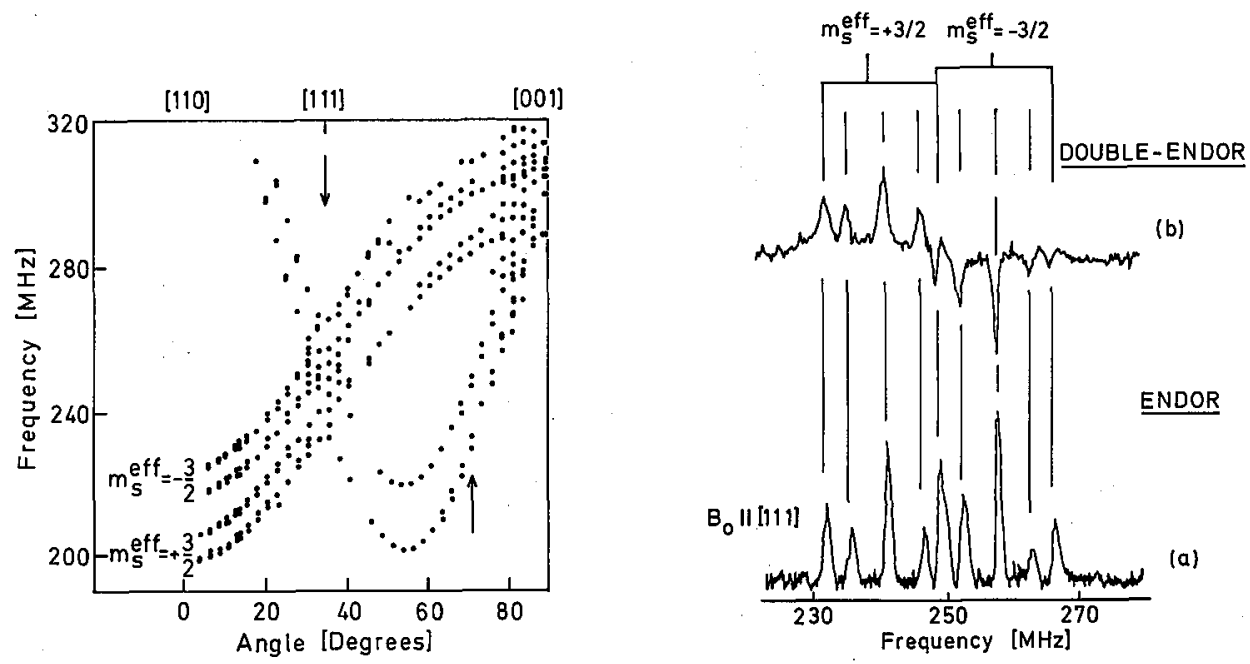

Fig. 8. The ENDOR spectrum for the Ga vacancy centre in GaP [10].

Fig. 9. The illustration of double ENDOR effect on the $\mathrm{Ga}$ vacancy in GaP. The double ENDOR effect has been observed for the indicated ENDOR line.

data obtained in this study is shown; as can be seen a small splitting of all the resonance lines is observed. Such splitting could originate from the lowering of the symmetry of the centre or from an additional hyperfine interaction within the 
same system. In order to decide between these two possibilities a double ENDOR experiment has been performed. The result of this experiment is shown in Fig. 9; the lower trace shows the conventional ENDOR spectrum as recorded for the [111] direction - indicated by an arrow in Fig. 8. The upper trace corresponds to a double ENDOR spectrum; the intensity of the most pronounced ENDOR line from the lower spectrum-indicated by an arrow-is followed as a function of the second rf frequency. As can be seen a (positive or negative) response is found for every ENDOR resonance proving that all transitions belong to the same system and ruling therefore out the possibility of a lower (though unresolved) symmetry type of the total EPR spectrum.

\section{References}

[1] G. Feher, Phys. Rev. 114, 1219 (1959).

[2] E.B. Hale, R.L. Mieher, Phys. Rev. 184, 739 (1969).

[3] D.A. van Wezep, C.A.J. Ammerlaan, Phys. Rev. B 37, 7268 (1988).

[4] D.A. van Wezep, C.A.J. Ammerlaan, Phys. Rev. B 32, 7129 (1985).

[5] H. E. Altink, to be published.

[6] J.R. Niklas, J.-M. Spaeth, Phys. Status Solidi B 101, 221 (1980).

[7] M. Sprenger, R. van Kemp, E.G. Sieverts, C.A.J. Ammerlaan, Phys. Rev. $B$ 35, 1582 (1987).

[8] T. Gregorkiewịcz, H.H.P.Th. Bekman, C.A.J. Ammerlaan, Phys. Rev. B 38, 3998 (1988).

[9] C.A.J. Ammerlaan, J.R. Niklas, in Proc. of Internat. Workshop on Hyperfine Interactions of Defects in Semiconductors, Cargese (France) 1990, to be published by North-Holland.

[10] J. Hage, J.R. Niklas, J.-M. Spaeth, Mater. Sci. Forum 10-12, 259 (1986). 